\title{
ON TITS' CENTRE CONJECTURE FOR FIXED POINT SUBCOMPLEXES
}

\author{
MICHAEL BATE, BENJAMIN MARTIN, AND GERHARD RÖHRLE
}

\begin{abstract}
We give a short and uniform proof of a special case of Tits' Centre Conjecture using a theorem of J-P. Serre $[8$ and a result from [1. We consider fixed point subcomplexes $X^{H}$ of the building $X=X(G)$ of a connected reductive algebraic group $G$, where $H$ is a subgroup of $G$.
\end{abstract}

\section{INTRODUCTION}

Let $G$ be a connected reductive linear algebraic group defined over an algebraically closed field $k$. Let $X=X(G)$ be the spherical Tits building of $G$, cf. [10]. Recall that the simplices in $X$ correspond to the parabolic subgroups of $G,[8, \S 3.1]$; for a parabolic subgroup $P$ of $G$, we let $x_{P}$ denote the corresponding simplex of $X$. The conjugation action of $G$ on itself naturally induces an action of $G$ on the building $X$, so the image of $G$ is a subgroup of the automorphism group of $X$. Given a subcomplex $Y$ of $X$, let $N_{G}(Y)$ denote the subgroup of $G$ consisting of elements which stabilize $Y$ (in this induced action).

Recall the geometric realization of $X$ as a bouquet of $n$-spheres. A subcomplex $Y$ of $X$ is called convex if whenever two points of $Y$ (in the geometric realization) are not opposite in $X$, then $Y$ contains the unique geodesic joining these points, [8, §2.1]. A convex subcomplex $Y$ of $X$ is contractible if it has the homotopy type of a point, [8, §2.2]. The following is a version due to J-P. Serre of the so-called "Centre Conjecture" by J. Tits, cf. [9, Lem. 1.2], [6, $\S 4],[8, \S 2.4]$, [11]. This has been proved by B. Mühlherr and J. Tits for spherical buildings of classical type [5]. The simplex referred to in the conjecture is called a centre for $Y$.

Conjecture 1.1. Let $Y$ be a convex contractible subcomplex of $X$. Then there is a simplex in $Y$ which is stabilized by all automorphisms of $X$ which stabilize $Y$.

For a subgroup $H$ of $G$ let $X^{H}$ be the fixed point subcomplex of the action of $H$, i.e., $X^{H}$ consists of the simplices $x_{P} \in X$ such that $H \subseteq P$. Thus, if $H \subseteq K \subseteq G$ are subgroups of $G$, then we have $X^{K} \subseteq X^{H}$; observe that $X^{\bar{H}}$ is always convex, cf. [8, Prop. 3.1]. Our main result, Theorem 3.1, gives a short, conceptual proof of a special case of Conjecture 1.1. namely, we consider subcomplexes of the form $Y=X^{H}$ for $H$ a subgroup of $G$, and we consider automorphisms only from $N_{G}(Y)$. The special case $G=\mathrm{GL}(V)$ in Theorem 3.1 generalizes the classical construction of upper and lower Loewy series, see Remark 3.2(ii).

The initial motivation for Tits' Conjecture 1.1 was a question about the existence of a certain parabolic subgroup associated with a unipotent subgroup of a Borel subgroup of $G$ (cf. [6, §4.1], [8, §2.4]). This existence theorem was ultimately proved by other means, [3, §3]. In Example 3.6 below we show that the existence of such a parabolic subgroup can be viewed as a special case of Theorem 3.1 .

2000 Mathematics Subject Classification. 51E24, 20E42, $20 \mathrm{G} 15$. 


\section{SERRE'S NOtion OF COMPLETE REDUCIBILITY}

Following Serre [8, Def. 2.2.1], we say that a convex subcomplex $Y$ of $X$ is $X$-completely reducible $(X$-cr $)$ if for every simplex $y \in Y$ there exists a simplex $y^{\prime} \in Y$ opposite to $y$ in $X$. The following is part of a theorem due to Serre, [6, Thm. 2]; see also [8, §2] and [11].

Theorem 2.1. Let $Y$ be a convex subcomplex of $X$. Then $Y$ is $X$-completely reducible if and only if $Y$ is not contractible.

The notion of convexity for subcomplexes of $X$ has the following nice characterization in terms of parabolic subgroups due to Serre, [8, Prop. 3.1].

Proposition 2.2. Let $Y$ be a subcomplex of $X$. Then $Y$ is convex if and only if whenever $P, P^{\prime}$, and $Q$ are parabolic subgroups in $G$ with $x_{P}, x_{P^{\prime}} \in Y$ and $Q \supseteq P \cap P^{\prime}$, then $x_{Q} \in Y$.

Note that many subcomplexes which arise naturally in the building are fixed point subcomplexes. For example, the apartments of $X$ are the subcomplexes $X^{T}$ for maximal tori $T$ of $G$ and, more generally, the smallest convex subcomplex containing two simplices $x_{P}$ and $x_{P^{\prime}}$ is $X^{P \cap P^{\prime}}$.

Following Serre [8, we say that a (closed) subgroup $H$ of $G$ is $G$-completely reducible ( $G$ cr) provided that whenever $H$ is contained in a parabolic subgroup $P$ of $G$, it is contained in a Levi subgroup of $P$; for an overview of this concept see for instance [7] and [8]. In the case $G=\mathrm{GL}(V)$ ( $V$ a finite-dimensional $k$-vector space) a subgroup $H$ is $G$-cr exactly when $V$ is a semisimple $H$-module, so this faithfully generalizes the notion of complete reducibility from representation theory. An important class of $G$-cr subgroups consists of those that are not contained in any proper parabolic subgroup of $G$ at all (they are trivially $G$-cr). Following Serre, we call them $G$-irreducible ( $G$-ir), [8]. As before, in the case $G=\operatorname{GL}(V)$, this concept coincides with the usual notion of irreducibility. If $H$ is a $G$-completely reducible subgroup of $G$, then $H^{0}$ is reductive, [7, Property 4].

Since $X^{H}$ is a convex subcomplex of $X=X(G)$ for any subgroup $H$ of $G$, Theorem 2.1 applies in this case and we have the following result (see [7, p19], [8, §3]):

Theorem 2.3. Let $H$ be a subgroup of $G$. Then $H$ is $G$-completely reducible if and only if the subcomplex $X^{H}$ is not contractible.

Remark 2.4. By convention, the empty subcomplex of $X$ is not contractible. This is consistent with Theorem 2.1, because $H$ is $G$-ir if and only if $X^{H}=\varnothing$, and a $G$-ir subgroup is $G$-cr.

Our next result [1, Thm. 3.10] gives an affirmative answer to a question by Serre, [7, p. 24]. The special case when $G=\mathrm{GL}(V)$ is just a particular instance of Clifford Theory.

Theorem 2.5. Let $N \subseteq H \subseteq G$ be subgroups of $G$ with $N$ normal in $H$. If $H$ is $G$-completely reducible, then so is $N$.

\section{Tits' Centre Conjecture for fixed point subcomplexes}

Here is the main result of this note.

Theorem 3.1. Let $Y$ be a convex, contractible subcomplex of $X$. Suppose that $Y$ is of the form $Y=X^{H}$ for a subgroup $H$ of $G$. Then there is a simplex in $Y$ which is stabilized by all elements in $N_{G}(Y)$. 
Proof. Let $M$ be the intersection of all parabolic subgroups of $G$ corresponding to simplices in $Y$. Since $H \subseteq M$, we have $X^{M} \subseteq X^{H}$. But every parabolic subgroup containing $H$ contains $M$, by definition of $M$. Hence $X^{M}=X^{H}$. Set $K:=N_{G}(Y)$. It is clear that $M$ is normal in $K$. Since $X^{K} \subseteq X^{M}$, it suffices to show that $X^{K} \neq \varnothing$. Now $Y=X^{M}$ is contractible, so Theorem 2.3 implies that $M$ is not $G$-cr. Thus, by Theorem 2.5, it follows that $K$ is not $G$-cr and again by Theorem 2.3 that $X^{K}$ is contractible. In particular, $X^{K}$ is non-empty, by Remark 2.4. Thus $K$ stabilizes a simplex in $X^{M}$, as claimed.

Remarks 3.2. (i). Let $H \subseteq K \subseteq G$ be subgroups of $G$ with $H$ normal in $K$. Suppose that $X^{H}$ is contractible. Since $H$ is normal in $K$, the latter permutes the simplices in $X^{H}$, and so $K \subseteq N_{G}\left(X^{H}\right)$. It thus follows from Theorem 3.1 that $K$ fixes a simplex in $X^{H}$.

(ii). Observe that Theorem 3.1 can be viewed as a generalization of the classical construction of upper and lower Loewy series in representation theory (for definitions, see e.g., [4]). Let $V$ be a finite-dimensional $k$-vector space. Let $H \subseteq K \subseteq \mathrm{GL}(V)$ be subgroups of $\mathrm{GL}(V)$ with $H$ normal in $K$ and suppose that $V$ is not $H$-semisimple. Then the upper and lower Loewy series of the $H$-module $V$ are proper $K$-stable flags in $V$, and so they provide "natural centres" for the action of $K$ on the complex $X(V)^{H}$, where $X(V)$ is the flag complex of $V$.

(iii). In [8, Prop. 2.11], J-P. Serre showed that Theorem 2.5 is a consequence of Tits' Centre Conjecture 1.1. So, Theorem 3.1 is just the reverse implication of Serre's result [8, Prop. 2.11] in the special case when Theorem 2.5 applies.

(iv). Let $k_{0}$ be any field and let $k$ be the algebraic closure of $k_{0}$. Suppose that $G$ is defined over $k_{0}$. One can define what it means for a subgroup $H$ defined over $k_{0}$ to be $G$-completely reducible over $k_{0}$, cf. [1, Sec. 5], [8, Sec. 3]. In [1, Thm. 5.8], it is proved that if $k_{0}$ is perfect, then a subgroup $H$ is $G$-cr over $k_{0}$ if and only if it is $G$-cr. Using this, one can show that the proof of Theorem 3.1 goes through for buildings of the form $X=X\left(G\left(k_{0}\right)\right)$. In particular, this includes many finite spherical buildings attached to finite groups of Lie type.

(v). In the Centre Conjecture 1.1, one considers all automorphisms of the building. If $X=X(G)$, then in many cases, Aut $X$ is generated by inner and graph automorphisms of $G$, together with field automorphisms (cf. [10, Intro.]). We will consider graph and field automorphisms in the setting of Theorem 3.1 in future work (see [2, Sec. 6]).

Our final result gives a characterization of subcomplexes of $X$ of the form $X^{H}$ for a subgroup $H$ of $G$.

Proposition 3.3. Let $Y \subseteq X$ be a subset of simplices of $X$. Then $Y$ is a subcomplex of $X$ of the form $Y=X^{H}$ for some subgroup $H$ of $G$ if and only if for every $n \in \mathbb{N}$, the following condition holds:

$$
\text { if } P_{1}, \ldots, P_{n}, Q \text { are parabolic subgroups with } x_{P_{i}} \in Y \text { and } Q \supseteq \bigcap_{i=1}^{n} P_{i} \text {, then } x_{Q} \in Y \text {. }
$$

Proof. First suppose that $Y=X^{H}$ for some subgroup $H$ of $G$. Let $n \in \mathbb{N}$ and let $x_{P_{1}}, \ldots, x_{P_{n}} \in Y$. If $Q$ is a parabolic subgroup of $G$ containing $\cap_{i=1}^{n} P_{i}$, then $Q$ contains $H$, because each $P_{i}$ does, so $x_{Q} \in Y$.

Conversely, suppose that condition (3.4) holds for all $n \in \mathbb{N}$. Let $H$ be the intersection of all $P$ such that $x_{P} \in Y$. By the descending chain condition, we have $H=\cap_{i=1}^{m} P_{i}$ for some $m \in \mathbb{N}$ and some $P_{i}$ with $x_{P_{i}} \in Y$. It follows from condition (3.4) for $n=m$ that for any parabolic subgroup $P$ containing $H, x_{P} \in Y$, so $X^{H} \subseteq Y$. It is clear from the definition of $H$ that $Y \subseteq X^{H}$. 
Remark 3.5. Note that $Y$ is a subcomplex of $X$ precisely when condition (3.4) holds for $n=1$. Further, by Proposition 2.2, $Y$ is convex if and only if condition (3.4) holds for $n=2$.

As indicated in the Introduction, a fundamental theorem of Borel and Tits on unipotent subgroups of Borel subgroups of $G$ [3, §3] yields a key example for Theorem 3.1 .

Example 3.6. Let $U$ be a non-trivial unipotent subgroup of $G$ contained in a Borel subgroup $B$ of $G$. Let $Y=X^{U}$. Note that $U$ is not $G$-cr; for if $U$ is contained in a Borel subgroup $B^{-}$opposite to $B$, then $U$ is contained in the maximal torus $B^{-} \cap B$ of $G$, which is absurd. So $Y$ is contractible, by Theorem 2.3 . Thus, by Theorem $3.1, N_{G}(U)$ stabilizes a simplex in $Y$, i.e., there is a parabolic subgroup $P$ of $G$ containing $N_{G}(U)$. Now, the construction of Borel and Tits in [3] yields such a parabolic subgroup $P$ which enjoys additional properties; for example, it is stabilized by automorphisms of $G$ which stabilize $U$. The framework for $G$-complete reducibility developed in [1] and subsequent papers allows one to associate such canonical parabolic subgroups to all non- $G$-cr subgroups of $G$, see [2, Sec. 5].

Acknowledgements: The authors acknowledge the financial support of EPSRC Grant EP/C542150/1 and Marsden Grant UOC0501. Part of the paper was written during a visit of the third author to the Max-Planck-Institute for Mathematics in Bonn.

\section{REFERENCES}

[1] M. Bate, B. Martin, G. Röhrle, A geometric approach to complete reducibility, Inv. math. 161, no. 1 (2005), 177-218.

[2] M. Bate, B. Martin, G. Röhrle, R. Tange, Closed orbits and uniform S-instability in Invariant Theory, preprint (2009).

[3] A. Borel, J. Tits, Éléments unipotents et sous-groupes paraboliques des groupes réductifs, I, Invent. Math. 12 (1971), 95-104.

[4] J. Dauns, Chains of modules with completely reducible quotients, Pacific J. Math. 17 (1966), 235-242.

[5] B. Mühlherr, J. Tits, The Centre Conjecture for non-exceptional buildings, J. Algebra 300 (2006), no. 2, 687-706.

[6] J-P. Serre, La notion de complète réductibilité dans les immeubles sphériques et les groupes réductifs, Séminaire au Collège de France, résumé dans [12, pp. 93-98], (1997).

[7] - The notion of complete reducibility in group theory, Moursund Lectures, Part II, University of Oregon, 1998, arXiv:math/0305257v1 [math.GR].

[8] __ Complète Réductibilité, Séminaire Bourbaki, 56ème année, 2003-2004, nº 932.

[9] J. Tits, Groupes semi-simples isotropes, Colloq. Théorie des Groupes Algébriques, Bruxelles, 1962.

[10] _ Buildings of spherical type and finite BN-pairs, Lecture Notes in Math. 386, Springer-Verlag (1974).

[11] Quelques cas d'existence d'un centre pour des ensembles de chambres qui sont convexes, non vides et ne contiennent pas de paires de chambres opposées, Séminaire au Collège de France, résumé dans [12, pp. 98-101], (1997).

[12] _ Théorie des groupes, Résumé des Cours et Travaux, Annuaire du Collège de France, $97^{\mathrm{e}}$ année, (1996-1997), 89-102. 
Department of Mathematics, University of York, York YO10 5DD, United Kingdom

E-mail address: meb505@york.ac.uk

Mathematics and Statistics Department, University of Canterbury, Private Bag 4800, Christchurch 8140, New Zealand

E-mail address: B.Martin@math.canterbury.ac.nz

Fakultät für Mathematik, Ruhr-Universität Bochum, D-44780 Bochum, Germany

E-mail address: gerhard.roehrle@rub.de 